\title{
PERITUBULAR MACROPHAGES AND SPERMATOGONIA ARE SEQUENTIALLY INCREASED IN THE TESTIS OF RATS AFTER MONO-(2-ETHYLHEXYL) PHTHALATE EXPOSURE
}

Running Title: Phthalates and Peritubular Macrophages

Summary Sentence: Phthalate-induced testicular injury results in an increase of specialized peritubular macrophages that may assist in the recovery of spermatogenesis.

Keywords: Environmental contaminants and toxicants, Immunology, Testis, Spermatogenesis

Ross Gillette $^{1 *}$, Richa Tiwary ${ }^{1 *}$, Jorine JLP Voss $^{1}$, Shavini N Hewage ${ }^{2}$, John H Richburg ${ }^{1 \dagger}$

${ }^{1}$ The Center for Molecular Carcinogenesis and Toxicology, Division of Pharmacology and Toxicology, College of Pharmacy, The University of Texas at Austin, Austin, TX, 78712, USA

${ }^{2}$ College of Natural Sciences, The University of Texas at Austin, Austin, TX, 78712, USA

Grant funding: Supported by NIH/NIEHS 5R01ES016591 and The Center for Molecular Carcinogenesis and Toxicology.

\section{Author Contributions}

Conceptualization: J.H.R., R.T., J.J.L.P.V.; Methodology: R.G., R.T., J.W.V.; Investigation: R.G., R.T., J.J.L.P.V., S.N.H.; Formal Analysis: R.G.; Writing - Original Draft: R.G.; Writing Review \& Editing: R.G., R.T., J.H.R.; Funding Acquisition: J.H.R.

Declaration of Interest: The authors declare no competing interests.

\footnotetext{
* These authors contributed equally to the work.

† Corresponding Author: john.richburg@austin.utexas.edu
} 


\section{Abstract}

Peripubertal exposure to the phthalate metabolite mono-(2-ethylhexyl) phthalate (MEHP)

3 in rodents causes testicular inflammation, spermatocyte apoptosis, and disruption of the blood-

4 testis barrier. The MEHP-induced inflammation response includes an infiltration of macrophages

5 and neutrophils to the testes, although the cause and purpose of this response is unknown.

6 Recently, a population of testicular macrophages phenotypically distinct from those resident in

7 the interstitium was described in mice. Testicular peritubular macrophages aggregate near the

8 spermatogonial stem cell niche and are believed to stimulate their differentiation. We

9 hypothesized that if testicular peritubular macrophages do indeed stimulate spermatogonial

10 differentiation, MEHP exposure would result in an increase of peritubular macrophages to

11 stimulate the replacement of lost spermatocytes. Male rats were exposed to $700 \mathrm{mg} / \mathrm{kg} \mathrm{MEHP}$ or

12 corn oil (vehicle control) via oral gavage at PND 28 and euthanized at 48 hours, 1 week, or 2

13 weeks later. Tubules were stained with immunofluorescent markers for macrophages and

14 undifferentiated spermatogonia. Peritubular macrophages were observed in rat testis similar to

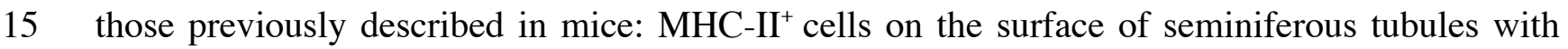

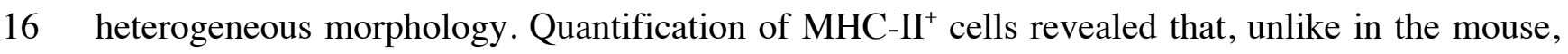

17 their numbers did not increase through puberty. MEHP increased macrophage presence by six-

18 fold 48-hours after exposure and remained elevated by two-fold two weeks after exposure. An

19 increase of differentiating spermatogonia occurred two weeks after MEHP exposure. Taken

20 together, our results suggest that peritubular macrophages play a crucial role in the testis

21 response to acute injury and the subsequent recovery of spermatogenesis. 


\section{INTRODUCTION}

Di-(2-ethylhexyl) phthalate (DEHP) is a ubiquitous plasticizer that is not chemically

24 bound to its substrates, which results in its release and eventual ingestion by humans and animals

25 [1,2]. Mono-(2-ethylhexyl) phthalate (MEHP) is the primary metabolite of DEHP and has been

26 observed at measurable concentrations in a majority of newborns [3] and in pre-pubertal children

27 from those observed in the US [4] and Germany [5]. MEHP is considered an endocrine

28 disrupting chemical (EDC) that is correlated with reduced free testosterone [6] and increased

29 prolactin and estradiol [7] in adult male humans. MEHP persists in measurable quantities in

30 semen [8] and urine and is correlated with a decrease in sperm quality [9]. Hence, one of the

31 primary concerns regarding MEHP exposure in humans is the deleterious effect it may have on

32 fertility and reproductive function.

33 Experimental animal models provide a majority of the mechanistic evidence that links

34 phthalate exposure to adverse fertility outcomes. Toxicant exposure, including MEHP, in the

35 testes primarily affect Sertoli cells [10], which sequester meiotic, and therefore antigenic, germ

36 cells behind a selective blood-testis barrier (BTB). The BTB protects germ cells from the

37 immune system, transfer necessary nutrients and information from the endocrine system, and

38 clean up apoptotic germ cells and waste products created during spermatogenesis (Reviewed in:

39 [11]). Acute high dose MEHP exposure in rodents results in the disruption of the Sertoli cell

40 cytoskeleton [12]. A secondary consequence of this disruption is the dysregulation of the

41 apoptosis inducing FAS/FAS-ligand system [13] which results in the widespread death of

42 maturing spermatocytes [14]. In addition, MEHP exposure causes disruption in the gap junctions

43 between Sertoli cells that form the BTB [15] which is, in part, regulated by sTNF $\alpha$ [16], a

44 compound that is capable of initiating an inflammatory immune response.

45 Indeed, a brief but large monocyte influx appears coincident with widespread 46 spermatocyte apoptosis following MEHP exposure [17]. This influx is at least in part elicited by 47 canonical pro-inflammatory cytokines (e.g. IL-1 $\alpha$, IL-6 and MCP-1) released by Sertoli cells and 48 not by spermatocyte apoptosis itself [18]. Although the infiltrating monocytes observed due to 
acute MEHP exposure are inflammatory in phenotype, they do not contribute to or exacerbate

50 spermatocyte apoptosis [19].

51 The purpose or function of the monocyte infiltration into the testes due to MEHP-

52 induced injury remains unknown. Macrophages, which mature from naïve monocytes, make up a

53 large portion of all cells in the testicular interstitium and are the most abundant immune cell type

54 in the testis [20]. Testicular macrophages can be broadly categorized as those resident to the

55 testes and those that infiltrate to the testes as a part of an inflammatory response. Resident

56 testicular macrophages perform a number of essential functions during development and

57 adulthood (Reviewed in: $[21,22]$ ). These functions include morphogenesis via vascularization

58 during organogenesis [23], assisting in the steroidogenesis of testosterone [24] and, therefore,

59 aiding spermatogenesis and maintaining an immunosuppressive environment $[25,26]$ presumably

60 to protect the integrity of the meiotic spermatogonial niche. Even under inflammatory conditions,

61 resident testicular macrophages have a unique response and tend to polarize towards an anti-

62 inflammatory phenotype; in response to LPS [25], bacteria [27], or IL-4 [28]. Recently, a novel

63 role for testicular macrophages was proposed in mice. A subset of macrophages were observed

64 on the surface of seminiferous tubules, concentrated in areas of undifferentiated spermatogonia,

65 and were found to aid in the differentiation of spermatogonia [29]. This specialized population of

66 macrophages, called peritubular macrophages, were found to be derived exclusively from

67 infiltrating populations of monocytes [30]. Therefore, we hypothesize that the monocyte

68 infiltration observed due to MEHP testicular damage may contribute to the seeding of peritubular

69 macrophages and aid in the recovery of the spermatogonial niche, rather than cause additional

70 damage as previously predicted. To test this hypothesis, we first evaluated if peritubular

71 macrophages are present in the rat testis, then describe their morphological and expression

72 phenotype, and, finally, determine if they associate with the spermatogonial niche. 
MATERials ANd Methods

\section{$74 \quad$ Animals and Housing}

All animal work was conducted using humane procedures that were preapproved by the

76 Institutional Animal Care and Use Committee at The University of Texas at Austin and followed

77 NIH guidelines (AUP-201900115). Males Fischer CDF344 rats were purchased from Harlan

78 (Indianapolis, IN) and shipped to The University of Texas at Austin at post-natal day (PND) 25.

79 Upon arrival, animals were maintained in a temperature-controlled housing facility on a 12:12

80 L:D cycle with ad libitum access to food and standard rat chow. After 3 days of habituation, on

81 PND 28, males were randomly assigned to one of two treatment exposure groups; vehicle control

82 (VEH) or MEHP.

83 Each animal was exposed via oral gavage to $700 \mathrm{mg} / \mathrm{kg}$ MEHP dissolved in corn oil or an

84 equivalent volume of corn oil (VEH). For the data shown in this paper, 7 animals were used in

85 each treatment group in 2 cohorts ( 3 animals per treatment per age in cohort 1 and 4 animals per

86 treatment per age in cohort 2). Following gavage, animals were checked for general health but

87 were otherwise left undisturbed in their home cage until sacrifice 48 hours after treatment, 1

88 week after treatment, or 2 weeks after treatment. Animals were euthanized by $\mathrm{CO}_{2}$ asphyxiation

89 followed by cervical dislocation. The testes were rapidly removed and weighed. One testis was

90 randomly selected for whole tubule immunofluorescence and was immediately placed in cold

91 PBS (Gibco, Cat\# 10010-023) on ice. The opposite testis was submerged in Bouin's Fixative on

92 a gentle rocker overnight. Bouin's was replaced with lithium-saturated ethanol the following day

93 and every subsequent day until the solution and the testis were clear.

94 Seminiferous tubules were isolated within 1-hr of sacrifice from testes that had been

95 placed in cold PBS on ice. The testis was decapsulated by puncturing and tearing the tunica and

96 the tubules evacuated into cold PBS. The tubules were gently teased apart and the interstitial

97 tissue removed with blunt forceps. The tubules were then washed 4 times in ice cold PBS to

98 further remove interstitial tissue and cells. The tubules were then fixed in $4 \%$ paraformaldehyde 
99 (Electron Microscopy Sciences, 15710-S) overnight at $4{ }^{\circ} \mathrm{C}$, washed an additional 4 times in cold 100 PBS, and stored in cold PBS at $4 \mathrm{C}$ until use.

101 Immunofluorescence staining was performed in 96 well plates on seminiferous tubules 102 with primary antibodies major histocompatibility complex class II [MHC Class II RT1D 103 (BioLegend, Clone OX-17, 205401, 1:200)], cluster of differentiation 68 [CD68 (Bio Rad, 104 MCA341R, 1:200)], cluster of differentiation 163 [CD163 (Invitrogen PA5-78961 \& MA5105 16656, Bio Rad, MCA34212, 1:200)], zinc finger and BTB domain-containing protein 16 106 [ZBTB16/PLZF (Santa Cruz Biotechnology, sc-22839, 1:200)] and secondary antibodies Alexa 107488 (Invitrogen, A11001, 1:500) and Alexa 568 (Invitrogen, A11036, 1:500). Hoescht 33342 108 (Life Technologies, H3570, 1:10000) was used as a DNA/nuclei counter stain. Stained tubules 109 were mounted whole on super frost slides with Flouromount-G (Southern Biotech, 0100-01) and 110 sealed with standard clear nail polish. Slides were stored at $-20^{\circ} \mathrm{C}$ until imaging.

111 Slides were imaged on a Zeiss scanning confocal microscope (Zeiss, LSM 710). Images 112 that were used to count macrophages and spermatogonia were taken at 20x $(425.10 \mu \mathrm{m}$ by $113425.10 \mu \mathrm{m})$ and with a depth of $15 \mathrm{um}$ in $3 \mathrm{um}$ increments to ensure the surface of the 114 seminiferous epithelium and spermatogonia were captured. Z-stacks were flattened in ImageJ 115 (version 1.49T) using the "Max Intensity" method. Positively stained cells in flattened images 116 were then counted with EBImage (version 4.24.0) and R (version 3.5.3). Cell count was 117 normalized to tubule area and are represented as cell counts per $10^{5}$ pixel area. An aggregate cell 118 count was calculated across 15 individual tubule observations within each animal by summing 119 the cell count and tubule area of each image and calculating the density of positive cells (\# 120 cells $/ 10^{5}$ area). This methodology considers each animal as the individual statistical unit rather 121 than each tubule observation as the individual statistical unit and avoids violations of non122 independence assumptions within statistical tests. Counts were intermittently verified by manual 123 counting in ImageJ to ensure accuracy. Cell counts were compared between treatments and 124 within age using a Wilcoxon Ranked-Sum test because the data were not normally distributed 125 and were considered significant if $\mathrm{p}<0.01$. Graphs were made in $\mathrm{R}$ with ggplot2 (version 3.1.1) 
and edited only for style in Adobe Illustrator (version CS5). Images for morphological and co-

127 staining analyses were taken at 63x (134.95 $\mu \mathrm{m}$ by $134.95 \mu \mathrm{m})$ at $\mathrm{z}$-depths that varied depending

128 on the depth of the cell being imaged.

\section{RESULTS}

130 Seminiferous tubules from PND30 Fischer CD344 male rats were separated from 131 interstitial tissue, subjected to immunofluorescence for MHCII, and mounted whole on slides for 132 analysis on a confocal microscope. A population of $\mathrm{MHCII}^{+}$cells was observed on the surface of 133 seminiferous tubules (20x - Fig 1A). Closer observation of these cells revealed irregular 134 morphology of the cell membrane and nucleus $(63 \mathrm{x}-$ Fig1B and $\mathbf{C}) . \mathrm{MHCII}^{+}$cells were sparse 135 and found on average once every 2 randomly captured $(425.1 \mu \mathrm{m}$ by $425.1 \mu \mathrm{m}$, average tubule 136 area $102,084 \mu^{2}$ ) windows. These cells were located on the surface of tubules often overlying 137 or nestled in-between, in the same plane, or in close proximity to the nuclei of peritubular myoid 138 cells (Fig $\mathbf{2 A} \& \mathbf{B}$ ), which were identified by their location and unique morphology. 139 Occasionally, long $(\sim 50-100 \mu \mathrm{m})$ protrusions originating from $\mathrm{MHCII}^{+}$cells appeared to extend 140 around or between the nuclei of peritubular myoid cells (Fig 2 C \& D). In rare instances the 141 nuclei of an $\mathrm{MHCII}^{+}$cell was observed under what appeared to be the nuclei of peritubular myoid 142 cells (Fig $\mathbf{2 E - H})$.

143 The morphology of $\mathrm{MHCII}^{+}$cells was heterogeneous. The majority of $\mathrm{MHCII}^{+}$cells 144 observed on the surface of the seminiferous tubule were either circular (Fig 3A) or spindeloid 145 (Fig 3B -- [31]) in morphology. Observation of $\mathrm{MHCII}^{+}$cells with elongated or stellate 146 morphology was rare, but was occasionally observed (Fig 3C and Fig 3D, respectively).

147 In order to determine if the $\mathrm{MHCII}^{+}$cells observed were in fact derived from the 148 monocyte lineage, tubules were double stained with $\mathrm{MHCII}^{+}$and the lysosomal protein CD68. 149 All observed $\mathrm{MHCII}^{+}$cells on the surface of seminiferous tubules were $\mathrm{CD} 8^{+}$regardless of the 150 morphology of $\mathrm{MHCII}^{+}$cells (Fig 4). All $\mathrm{MHCII}^{+}$cells remained $\mathrm{CD}^{+} 8^{+}$regardless of the age or 151 treatment of the animal analyzed (PND30; Fig 4A-B, PND 35; Fig 4C-D, PND 42; Fig 4E-F). 
We hypothesized that if the function of peritubular macrophages is indeed to stimulate

153 the differentiation of spermatogonial cells, then depletion of maturing germ cells via MEHP

154 treatment would induce the mobilization of peritubular macrophages to the seminiferous tubules.

155 PND 28 male rats were exposed to either MEHP or corn oil (VEH) and sacrificed after 48 hours,

1561 week, or 2 weeks with no further manipulation and the number of $\mathrm{MHCII}^{+}$cells, controlled for

157 by tubule area, was counted $(n=7$ per treatment per age - Fig 5). After 48 hours, the population

158 of $\mathrm{MHCII}^{+}$was significantly elevated $\sim 6.5$-fold compared to $\mathrm{VEH}$ males of the same age $(\mathrm{U}=3$,

$159 \mathrm{p}=0.002-$ Fig 6). The number of $\mathrm{MHCII}^{+}$cells began to decline but remained significantly

160 elevated in MEHP animals $\sim 6$ fold after 1 week $(U=0, p=0.0003)$ and $\sim 2.5$-fold after 2 weeks

$161(\mathrm{U}=6, \mathrm{p}=0.005)$. Age did not affect the number of $\mathrm{MHCII}^{+}$cells in $\mathrm{VEH}$ animals as determined

162 by a Kruskal-Wallis test $(\mathrm{H}(2)=4.1039, \mathrm{p}=0.1285)$. Subsequent pairwise Kruskal-Wallis rank

163 sum tests, adjusted with the Benjamini-Hochberg method, also failed to identify any differences

164 in $\mathrm{MHCII}^{+}$cell counts across age and within VEH animals.

In order to determine the distribution of $\mathrm{MHCII}^{+}$cells, a density analysis of the number of

$166 \mathrm{MHCII}^{+}$cells per tubule area was performed (Fig 7). Males exposed to the VEH treatment,

167 which represent a "normal" developmental trajectory, at the 48 hour and 1 week time point had a

168 large proportion of instances $(\sim 75 \%)$ that had no $\mathrm{MHCII}^{+}$cells. The number of instances with

169 zero $\mathrm{MHCII}^{+}$cells was reduced to $56 \%$ in $\mathrm{VEH}$ treated males at 2 week time point, which

170 suggests that while the total number of $\mathrm{MHCII}^{+}$doesn't change with age, their localization does.

171 The distribution of $\mathrm{MHCII}^{+}$cells in MEHP treated males was markedly different. Instances with

172 no $\mathrm{MHCII}^{+}$cells was only $32 \%$ at 48 hours and 2 weeks and was slightly decreased at the 1 week

173 time point (25\%). There were isolated observations of high-density concentrations of $\mathrm{MHCII}^{+}$

174 cells in MEHP treated animals (>8 $\mathrm{MHCII}^{+}$cells per $10^{5}$ area $-12 \%$ at $48 \mathrm{hrs}$ and $1 \%$ at 1 week)

175 but none in the VEH treated groups. The majority of the increase in $\mathrm{MHCII}^{+}$cells seen due to

176 MEHP treatment was observed in intermediate densities ( $<8 \mathrm{MHCII}^{+}$cells per $10^{5}$ area).

177 Finally, we stained whole tubules with PLZF to determine if spermatogonial number was

178 affected by MEHP treatment. We found no difference in the number of PLZF ${ }^{+}$cells at either the 
48-hour or 1 week time point. However, the number of $\mathrm{PLZF}^{+}$cells was increased due to MEHP exposure at the 2-week time point $(\mathrm{U}=3, \mathrm{p}<0.001-$ Fig 8).

\section{DISCUSSION}

Testicular macrophages perform an eclectic set of functions in the testes that span, and

184 are essential to, the life cycle (Reviewed in: [22]). During development, testicular macrophages

185 aid in the organogenesis of the testis by directing vascularization [23]. During adolescence, 186 testicular macrophages stimulate the differentiation and proliferation of Leydig cells, the prime 187 steroid-hormone producing cell in the testis [32]. In adulthood, testicular macrophages aid in the 188 ultimate role of the testes, spermatogenesis, by aiding in the steroidogenesis of testosterone [24]. 189 Throughout the lifespan, testicular macrophages help maintain an immune-privileged

190 environment in the testis and therefore they protect sanctity of spermatogenesis [33]. Recently, a 191 newly discovered function was ascribed to testicular macrophages. A subset of testicular 192 macrophages (peritubular macrophages) was identified in mice that localize to the surface of 193 seminiferous tubules near the spermatogonial niche that when depleted disrupts spermatogonial 194 differentiation [29].

195 The current work aimed to describe the peritubular subset of testicular macrophages in 196 the rat for the first time, characterize their morphology and distribution, and observe their newly 197 ascribed function in an injury model. While previous models have shown that depleting 198 macrophages from the testis reduces the number of differentiating spermatogonia [29], here we

199 demonstrate that depletion of spermatocytes by exposure to MEHP mobilizes peritubular 200 macrophages to the surface of seminiferous tubules in large numbers for at least 2 weeks. This 201 increase is concurrent with an increase in the number of differentiating spermatogonia. This 202 work is significant in that it is one of the first demonstrations that peritubular macrophages are 203 specifically mobilized by testicular injury and the large-scale loss of spermatocytes. 
MEHP is a well described Sertoli cell toxicant [12], which causes widespread loss of

205 spermatocytes [14]. The loss of spermatocytes due to MEHP exposure is age- (younger $>$ older),

206 species- (rat $>$ mouse), and dose-dependent [17]. The loss of spermatocytes is concurrent with a

207 massive but transient ( 48 hours) monocyte influx that is congruent with the degree of

208 spermatocyte loss; the monocyte influx is more robust in younger animals, larger in rats

209 compared to mice and larger at higher doses of MEHP [17]. It was initially hypothesized that

210 because the monocyte influx induced by MEHP was proportional to the loss of spermatocytes,

211 this large-scale inflammatory immune response might exacerbate, or be the cause of, the loss of

212 spermatocytes and thus account for the specificity of the observed effects. This was found not to

213 be the case. The depletion of circulating monocytes, which neutralizes the MEHP induced

214 monocyte influx, has no effect on spermatocyte apoptosis [19]. The precipitating cause and the

215 functional purpose of this influx is not yet fully understood, but our current results suggest that at

216 least one consequence is an increase in the long-term (2 week) presence of peritubular

217 macrophages.

218 Given that the only known function of peritubular macrophages is to stimulate the

219 differentiation of spermatogonia, and that a loss of spermatocytes would necessitate their

220 replacement by the induction of spermatogonial differentiation, it is reasonable to suggest that

221 the increase in peritubular macrophages we observe is to stimulate spermatogonial differentiation

222 to replace the lost spermatocytes. Our data indirectly support this hypothesis; 2 weeks after

223 MEHP exposure, a time point where peritubular macrophages remain elevated, the number of

$224 \mathrm{PLZF}^{+}$cells (differentiating spermatogonia) is increased. This hypothesis requires additional

225 evidence and experiments, which are currently underway in our laboratory.

226 Peritubular macrophages may, of course, have functions other than, or in addition to, the

227 stimulation of spermatogonial differentiation. There are two primary immunoreactivity profiles

228 of macrophages that populate the testes in rats; tissue resident testicular-macrophages (CD68

$\left.229 / \mathrm{CD} 63^{+}\right)$and newly arrived monocyte-like testicular macrophages (CD68 $\left.{ }^{+} / \mathrm{CD} 163^{-}\right)($Reviewed

230 in: [21]). These two populations have distinct expression phenotypes that represent anti- 
231 inflammatory tissue resident macrophages $\left(\mathrm{CD}^{\circ} / \mathrm{CD} 163^{+}\right)$and "alternatively activated 232 macrophages" with suppressed inflammatory activity (CD68 $/$ CD163) [28].

233 We found that all peritubular macrophages in rats are CD68 ${ }^{+}$, indicating that they are 234 either derived from circulating monocytes that quickly (within 48 hours) transition and localize 235 to the seminiferous tubules or they constantly maintain the $\mathrm{CD}^{+} 8^{+}$phenotype regardless of when 236 they arrived in the testes. It is noteworthy that all peritubular macrophages in VEH animals were 237 also $\mathrm{CD}^{+} 8^{+}$at all time points, which supports the latter possibility; they maintain a $\mathrm{CD}^{+}$ 238 phenotype. This is consistent with previous observations in mice that found macrophages are 239 exclusively seeded from bone-marrow derived circulating monocytes using lineage-tracing 240 techniques [30]. Determining a definitive immunoreactive profile for peritubular macrophages in 241 future experiments is essential so that further characterization can be performed (e.g. fluorescent 242 activated cell sorting to allow for macrophage separation and transcriptome profiling). Efforts 243 are currently underway to definitively describe the CD163 phenotype of peritubular 244 macrophages in rats.

245 The location and morphology of peritubular macrophages also provide some evidence of 246 their possible function. Sertoli cells can perform macrophage-like functions and therefore serve 247 an immune cell role in the seminiferous epithelium; phagocytosis and ingestion of apoptotic 248 spermatocytes [34] and microbe detection via the Toll-like receptor [35]. Sertoli cells actively 249 participate in the clearance of apoptotic spermatocytes [36] and secrete the powerful monocyte 250 chemoattractant protein-1 (MCP-1) [18] specifically due to MEHP induced injury. Under normal 251 physiological conditions, Sertoli cells can induce germ cell apoptosis and ingest said cells such 252 that the meiotic and immunogenic remnants remain sequestered behind the blood-testis-barrier 253 (Reviewed in: [37]). It is, however, possible that in extreme cases of spermatocyte loss, such as 254 those induced by acute MEHP exposure, Sertoli cells are not capable of keeping up with the 255 processing of apoptotic bodies. A possible consequence of this scenario is that if the blood-testis256 barrier becomes compromised, an effect observed due to MEHP exposure [15], an auto-antigenic 257 immune response could be mounted against the "non-self" antigens and cause further damage. 
258 Sertoli cells can act as antigen presenting cells which diminishes inflammatory immune 259 responses [38]. That peritubular macrophages reside in such close proximity to Sertoli cells, that 260 they are themselves antigen presenting cells, and that they respond specifically to an event with 261 widespread apoptosis, suggests that they may play a role in the tolerization of inflammatory 262 immune responses in certain catastrophic scenarios. Direct macrophage cell-cell contact and 263 communication in the testis is not unheard of. Interstitial macrophages are known to directly 264 communicate with Leydig cells and aid in steroidogenesis [39]. That peritubular macrophages 265 could contact and interact with Sertoli cells would not be unlikely.

266 The work presented here demonstrates that a specialized sub-category of testicular 267 macrophage infiltrates and occupies the testes due to acute testicular injury in rats. This work is 268 fundamental to understanding the mechanisms by which infertility and azoospermia occur in 269 adult males. Correlational observations in humans consistently note an increased presence of $270 \mathrm{CD}^{+} 8^{+}$macrophages in infertile men [40,41]. These studies suggest the presence of $\mathrm{CD}^{+}$ 271 macrophages may contribute to or be the cause of infertility. These studies, however, are 272 retrospective in nature and may misinterpret the significance of the increased presence of 273 macrophages. Although more experimentation is needed, our data, and that of our predecessors, 274 suggests instead that $\mathrm{CD}^{+} 8^{+}$peritubular macrophages initially infiltrate the testis because of the 275 loss of maturing spermatocytes and to stimulate spermatogonial differentiation. 


\section{REFERENCES}

278 [1] Takehisa H, Naoko E, Masahiko S, Katsuhide T, Moriyuki O, Keizoh S, Mutsuko T, Kenji K, Shin'ichiro N, Toshio O. Release behavior of diethylhexyl phthalate from the polyvinyl-chloride tubing used for intravenous administration and the plasticized PVC

[2] Hanawa T, Muramatsu E, Asakawa K, Suzuki M, Tanaka M, Kawano K, Seki T, Juni K, Nakajima S. Investigation of the release behavior of diethylhexyl phthalate from the polyvinyl-chloride tubing for intravenous administration. International Journal of Pharmaceutics 2000; 210:109-115.

[3] Latini G, De Felice C, Presta G, Del Vecchio A, Paris I, Ruggieri F, Mazzeo P. In utero exposure to di-(2-ethylhexyl)phthalate and duration of human pregnancy. Environ Health Perspect 2003; 111:1783-1785.

[4] Silva MJ, Barr DB, Reidy JA, Malek NA, Hodge CC, Caudill SP, Brock JW, Needham LL, Calafat AM. Urinary levels of seven phthalate metabolites in the U.S. population from the National Health and Nutrition Examination Survey (NHANES) 1999-2000. Environ Health Perspect 2004; 112:331-338.

[5] Becker K, Seiwert M, Angerer J, Heger W, Koch HM, Nagorka R, Roßkamp E, Schlüter C, Seifert B, Ullrich D. DEHP metabolites in urine of children and DEHP in house dust. Int J Hyg Environ Health 2004; 207:409-417.

[6] Pan G, Hanaoka T, Yoshimura M, Zhang S, Wang P, Tsukino H, Inoue K, Nakazawa H, Tsugane S, Takahashi K. Decreased serum free testosterone in workers exposed to high levels of di-n-butyl phthalate (DBP) and di-2-ethylhexyl phthalate (DEHP): a crosssectional study in China. Environ Health Perspect 2006; 114:1643-1648.

[7] Li S, Dai J, Zhang L, Zhang J, Zhang Z, Chen B. An association of elevated serum prolactin with phthalate exposure in adult men. Biomed Environ Sci 2011; 24:31-39.

[8] Han SW, Lee H, Han SY, Lim DS, Jung KK, Kwack SJ, Kim KB, Lee BM. An exposure assessment of di-(2-ethylhexyl) phthalate (DEHP) and di-n-butyl phthalate (DBP) in human semen. J Toxicol Environ Health Part A 2009; 72:1463-1469.

[9] Wang Y-X, You L, Zeng Q, Sun Y, Huang Y-H, Wang C, Wang P, Cao W-C, Yang P, Li Y-F, Lu W-Q. Phthalate exposure and human semen quality: Results from an infertility clinic in China. Environ Res 2015; 142:1-9.

[10] Boekelheide K, Neely MD, Sioussat TM. The Sertoli cell cytoskeleton: a target for toxicant-induced germ cell loss. Toxicol Appl Pharmacol 1989; 101:373-389.

[11] França LR, Hess RA, Dufour JM, Hofmann MC, Griswold MD. The Sertoli cell: one hundred fifty years of beauty and plasticity. vol. 4. John Wiley \& Sons, Ltd (10.1111); 2016.

[12] Richburg JH, Boekelheide K. Mono-(2-ethylhexyl) phthalate rapidly alters both Sertoli cell vimentin filaments and germ cell apoptosis in young rat testes. Toxicol Appl Pharmacol 1996; 137:42-50.

[13] Lee J, Richburg JH, Shipp EB, Meistrich ML, Boekelheide K. The Fas system, a regulator of testicular germ cell apoptosis, is differentially up-regulated in Sertoli cell versus germ cell injury of the testis. Endocrinology 1999; 140:852-858.

[14] Richburg JH, Nañez A, Gao H. Participation of the Fas-signaling system in the initiation of germ cell apoptosis in young rat testes after exposure to mono-(2-ethylhexyl) phthalate. Toxicol Appl Pharmacol 1999; 160:271-278.

[15] Yao P-L, Lin Y-C, Richburg JH. Mono-(2-ethylhexyl) phthalate-induced disruption of 
junctional complexes in the seminiferous epithelium of the rodent testis is mediated by MMP2. Biology of Reproduction 2010; 82:516-527.

[16] Yao P-L, Lin Y-C, Sawhney P, Richburg JH. Transcriptional regulation of FasL expression and participation of sTNF-alpha in response to sertoli cell injury. J Biol Chem 2007; 282:5420-5431.

[17] Murphy CJ, Stermer AR, Richburg JH. Age- and Species-Dependent Infiltration of Macrophages into the Testis of Rats and Mice Exposed to Mono-(2-Ethylhexyl) Phthalate (MEHP)1. Biology of Reproduction 2014; 91:247-11.

[18] Stermer AR, Murphy CJ, Ghaffari R, Di Bona KR, Voss JJ, Richburg JH. Mono-(2ethylhexyl) phthalate-induced Sertoli cell injury stimulates the production of proinflammatory cytokines in Fischer 344 rats. Reproductive Toxicology 2017; 69:150_ 158.

[19] Voss JJLP, Stermer AR, Ghaffari R, Tiwary R, Richburg JH. MEHP-induced rat testicular inflammation does not exacerbate germ cell apoptosis. Reproduction 2018; 156:35-46.

[20] Niemi M, Sharpe RM, Brown WR. Macrophages in the interstitial tissue of the rat testis. Cell Tissue Res 1986; 243:337-344.

[21] Hedger MP. Macrophages and the immune responsiveness of the testis. J Reprod Immunol 2002; 57:19-34.

[22] Bhushan S, Meinhardt A. The macrophages in testis function. J Reprod Immunol 2017; 119:107-112.

[23] DeFalco T, Bhattacharya I, Williams AV, Sams DM, Capel B. Yolk-sac-derived macrophages regulate fetal testis vascularization and morphogenesis. Proc Natl Acad Sci USa 2014; 111:E2384-93.

[24] Hutson JC. Development of cytoplasmic digitations between Leydig cells and testicular macrophages of the rat. Cell Tissue Res 1992; 267:385-389.

[25] Kern S, Robertson SA, Mau VJ, Maddocks S. Cytokine secretion by macrophages in the rat testis. Biology of Reproduction 1995; 53:1407-1416.

[26] Hayes R, Chalmers SA, Nikolic-Paterson DJ, Atkins RC, Hedger MP. Secretion of bioactive interleukin 1 by rat testicular macrophages in vitro. J Androl 1996; 17:41-49.

[27] Bhushan S, Hossain H, Lu Y, Geisler A, Tchatalbachev S, Mikulski Z, Schuler G, Klug J, Pilatz A, Wagenlehner F, Chakraborty T, Meinhardt A. Uropathogenic E. coli induce different immune response in testicular and peritoneal macrophages: implications for testicular immune privilege. PLoS ONE 2011; 6:e28452.

[28] Winnall WR, Muir JA, Hedger MP. Rat resident testicular macrophages have an alternatively activated phenotype and constitutively produce interleukin-10 in vitro. $\mathrm{J}$ Leukoc Biol 2011; 90:133-143.

[29] DeFalco T, Potter SJ, Williams AV, Waller B, Kan MJ, Capel B. Macrophages Contribute to the Spermatogonial Niche in the Adult Testis. Cell Rep 2015; 12:11071119.

[30] Mossadegh-Keller N, Gentek R, Gimenez G, Bigot S, Mailfert S, Sieweke MH. Developmental origin and maintenance of distinct testicular macrophage populations. $\mathbf{J}$ Exp Med 2017; 214:2829-2841.

[31] Heinrich F, Lehmbecker A, Raddatz BB, Kegler K, Tipold A, Stein VM, Kalkuhl A, Deschl U, Baumgärtner W, Ulrich R, Spitzbarth I. Morphologic, phenotypic, and transcriptomic characterization of classically and alternatively activated canine blood- 
derived macrophages in vitro. PLoS ONE 2017; 12:e0183572.

[32] Gaytan F, Bellido C, Aguilar E, van Rooijen N. Requirement for testicular macrophages in Leydig cell proliferation and differentiation during prepubertal development in rats. J Reprod Fertil 1994; 102:393-399.

[33] Kern S, Maddocks S. Indomethacin blocks the immunosuppressive activity of rat testicular macrophages cultured in vitro. J Reprod Immunol 1995; 28:189-201.

[34] Nakanishi Y, Shiratsuchi A. Phagocytic removal of apoptotic spermatogenic cells by Sertoli cells: mechanisms and consequences. Biol Pharm Bull 2004; 27:13-16.

[35] Riccioli A, Starace D, Galli R, Fuso A, Scarpa S, Palombi F, De Cesaris P, Ziparo E, Filippini A. Sertoli cells initiate testicular innate immune responses through TLR activation. JI 2006; 177:7122-7130.

[36] Tay TW, Andriana BB, Ishii M, Choi EK, Zhu XB, Alam MS, Tsunekawa N, Kanai Y,

[37] Print CG, Loveland KL. Germ cell suicide: new insights into apoptosis during Kurohmaru M. Phagocytosis plays an important role in clearing dead cells caused by mono(2-ethylhexyl) phthalate administration. Tissue Cell 2007; 39:241-246.

[39] Hutson JC. Changes in the Concentration and Size of Testicular Macrophages during spermatogenesis. Bioessays 2000; 22:423-430. 
Figure 1. A) $\mathrm{MHCII}^{+}$peritubular macrophages (arrow heads) are shown on the surface of a whole seminiferous tubule at 20x; scale bar $=100 \mu \mathrm{m}$. B-C) Up close $(63 \mathrm{x}$; scale bar $=25 \mu \mathrm{m})$ confocal images show two $\mathrm{MHCII}^{+}$peritubular macrophages with heterogeneous morphology on the surface of a seminiferous tubule.

Figure 2. A-B) Two separate macrophages are shown at high magnification (63x; scale bar $=25$ $\mu \mathrm{m})$ nestled between the nuclei of peritubular myoid cells. The macrophage cell surface marker MHCII can clearly be seen to occupy exclusive space in the same plane and between the peritubular myoid cell nuclei. C-D) Long (>100 $\mu \mathrm{m})$ extensions can be seen protruding bidirectionally or unidirectionally (respectively) from $\mathrm{MHCII}^{+}$cells, sometimes meandering under the nuclei of other cells $(63 \mathrm{x}$; scale bar $=25 \mu \mathrm{m})$. E-F) An $\mathrm{MHCII}^{+}$cell is shown in two different scanning planes $(\mathrm{E}=\mathrm{Z}$-Depth $2.324 \mu \mathrm{m}$ and $\mathrm{F}=\mathrm{Z}$-Depth $4.316 \mu \mathrm{m})$ that is nestled between three peritubular myoid cell nuclei and under the nuclei of two cells that appear to also be peritubular myoid cells $(63 \mathrm{x}$; scale bar $=50 \mu \mathrm{m})$. G) A 3D brightness histogram and $\mathrm{H}$ ) a reconstructed $3 \mathrm{D}$ image clearly show an $\mathrm{MHCII}^{+}$cell under the nuclei of what appear to be peritubular myoid cells.

Figure 3. The various morphologies of $\mathrm{MHCII}^{+}$peritubular macrophages observed on the surface of seminiferous tubules is shown; A) circular $(63 \mathrm{x}$; scale bar $=25 \mu \mathrm{m})$, B) spindeloid $(63 x$; scale bar $=25 \mu \mathrm{m}), C)$ elongated $(63 x$; scale bar $=50 \mu \mathrm{m}), \mathrm{D})$ stellate $(63 \mathrm{x}$; scale bar $=25$ $\mu \mathrm{m})$.

Figure 4. Dual staining of peritubular macrophages with MHCII (green) and CD68 (red) are shown at all treatment and age endpoints; A-B) 48 hours after MEHP exposure, C-D) 1 week after MEHP exposure, and E-F) 2 weeks after MEHP exposure. The Hoescht (blue) nuclei counter stain is used in all images and the scale bar shown in part $\mathrm{A}$ is the same for all images. All $\mathrm{MHCII}^{+}$macrophages are also $\mathrm{CD} 68^{+}$regardless of treatment, age, or time after exposure. All imaged were taken at $63 \mathrm{x}$. The scale bar $(50 \mu \mathrm{m})$ represented in panel A applies to all images in this figure.

Figure 5. Representative micrographs of $\mathrm{MHCII}^{+}$(green) peritubular macrophages are shown on the surface of seminiferous tubules in close proximity to $\mathrm{PLZF}^{+}$(red) spermatogonia in both VEH and MEHP treated rats. At 48 hours after exposure, dense clusters of primarily circular $\mathrm{MHCII}^{+}$cells are observed on tubules with more single $\mathrm{PLZF}^{+}$cells. Circular $\mathrm{MHCII}^{+}$cells are not seen in $\mathrm{VEH}$ treated or 1 week/2 week MEHP treated samples and the maximum density of $\mathrm{MHCII}^{+}$cells decreases at 1week/2 weeks after MEHP exposure. All images in this figure were taken at 20x. The scale bar $(50 \mu \mathrm{m})$ represented in the top left panel applies to all images in this figure.

Figure 6. The number of $\mathrm{MHCII}^{+}$cells, normalized to tubule area, is shown. In VEH treated males, the number of peritubular macrophages does not increase through puberty as previously reported in mice, but stays at a constant low level. At all time points, the number of $\mathrm{MHCII}^{+}$cells on the surface of seminiferous tubules is elevated in males exposed to MEHP; $* \mathrm{p}<0.01$ and ** $\mathrm{p}<0.001$. 
Figure 7. The proportion of individual observations as a function of density (\# MHCII ${ }^{+}$cells $/ 10^{5}$ tubule area) is shown. Observations in VEH treated animals show a majority $(\sim 75 \%)$ of instances with no $\mathrm{MHCII}^{+}$cells at 48 hours and 1 week after treatment, which is diminished at 2 weeks. In MEHP treated males, the proportion of tubule observations with no $\mathrm{MHCII}^{+}$cells is lower $(\sim 30-$ $25 \%)$. Although high densities of $\mathrm{MHCII}^{+}$cells were observed in MEHP treated males, the majority of the observed increase was due to moderate densities.

Figure 8. The number of $\mathrm{PLZF}^{+}$spermatogonia in VEH and MEHP treated male rats is shown normalized to tubule area. The $\mathrm{PLZF}^{+}$count appears to diminish with age, but is primarily a factor of area normalization, which is attributed to the expansion of tubules as spermatogenesis progresses through puberty. MEHP treated males show an increase $(* * \mathrm{p}<0.001)$ of $\mathrm{PLZF}^{+}$ spermatogonia 2 weeks after exposure. 


\section{FigureS}

\section{Figure 1}
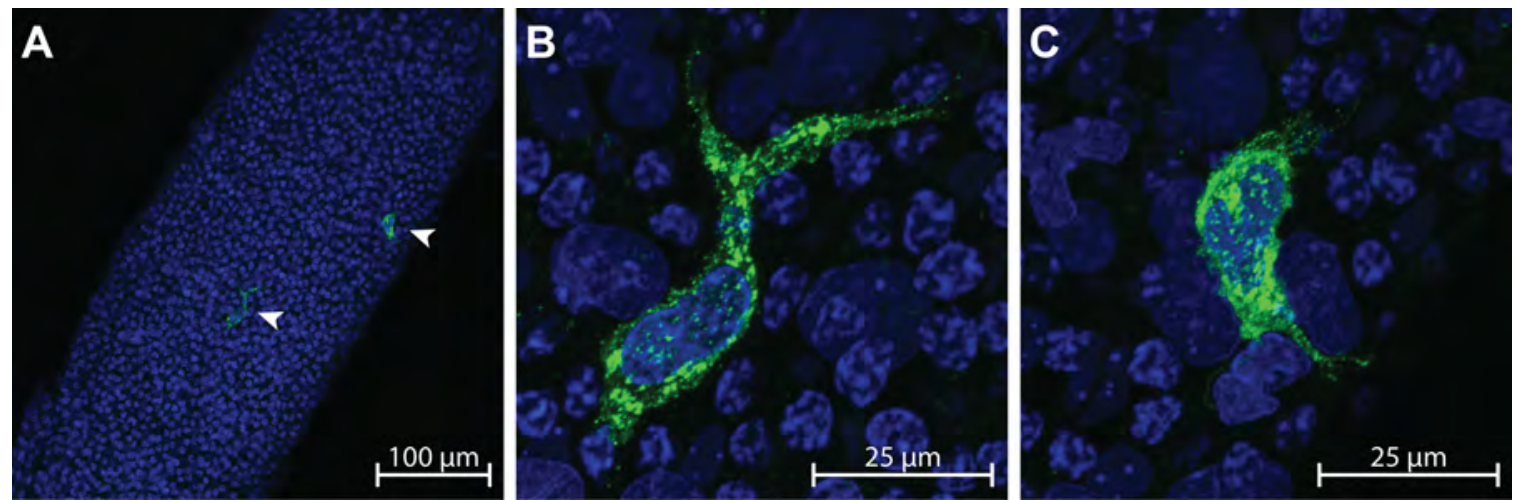

461 


\section{Figure 2}
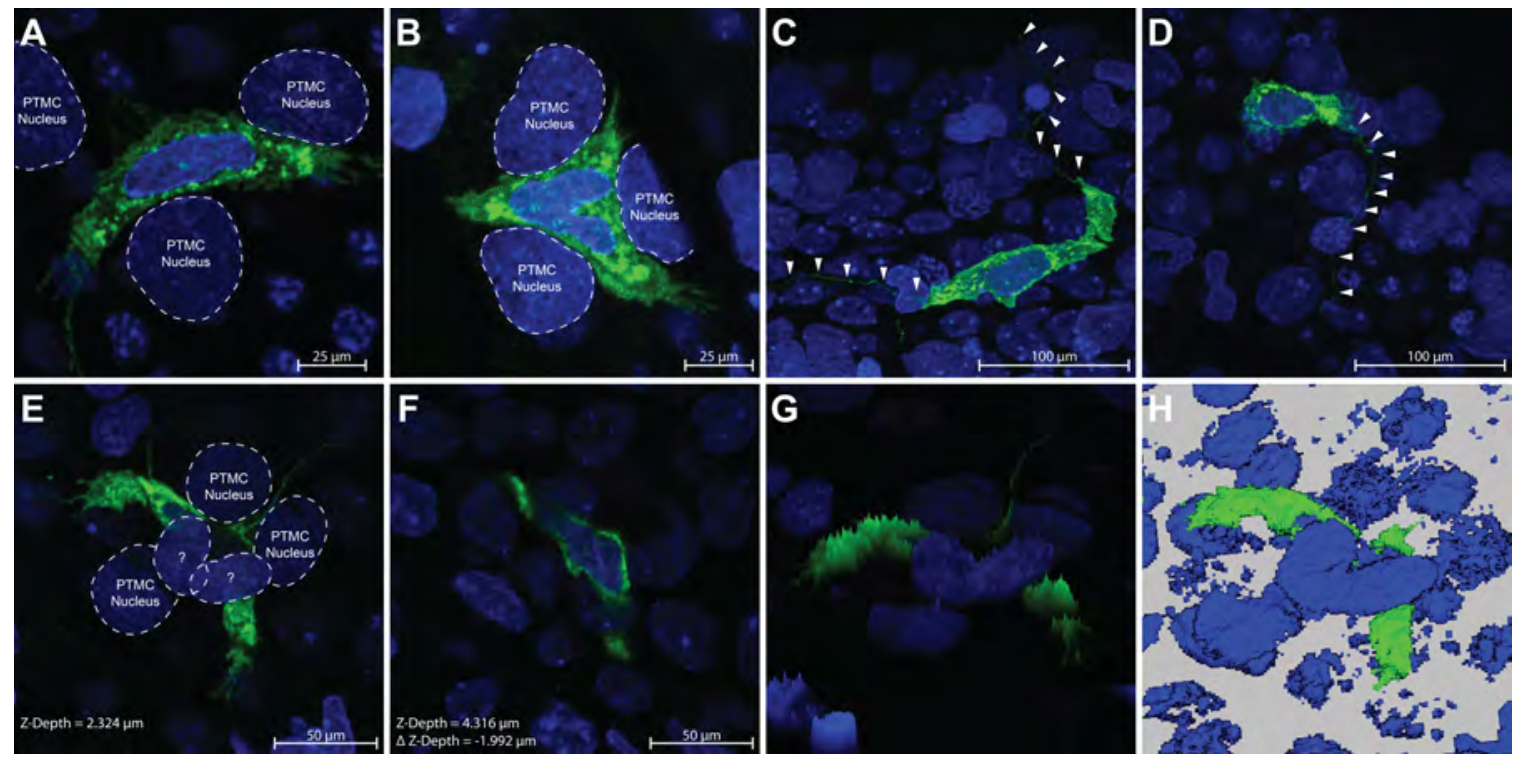

464 


\section{Figure 3}
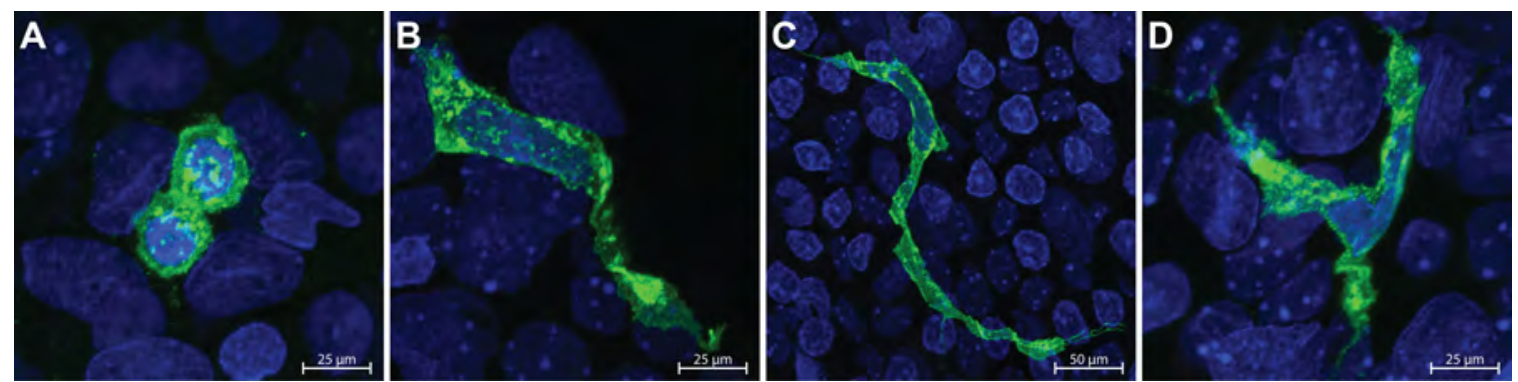

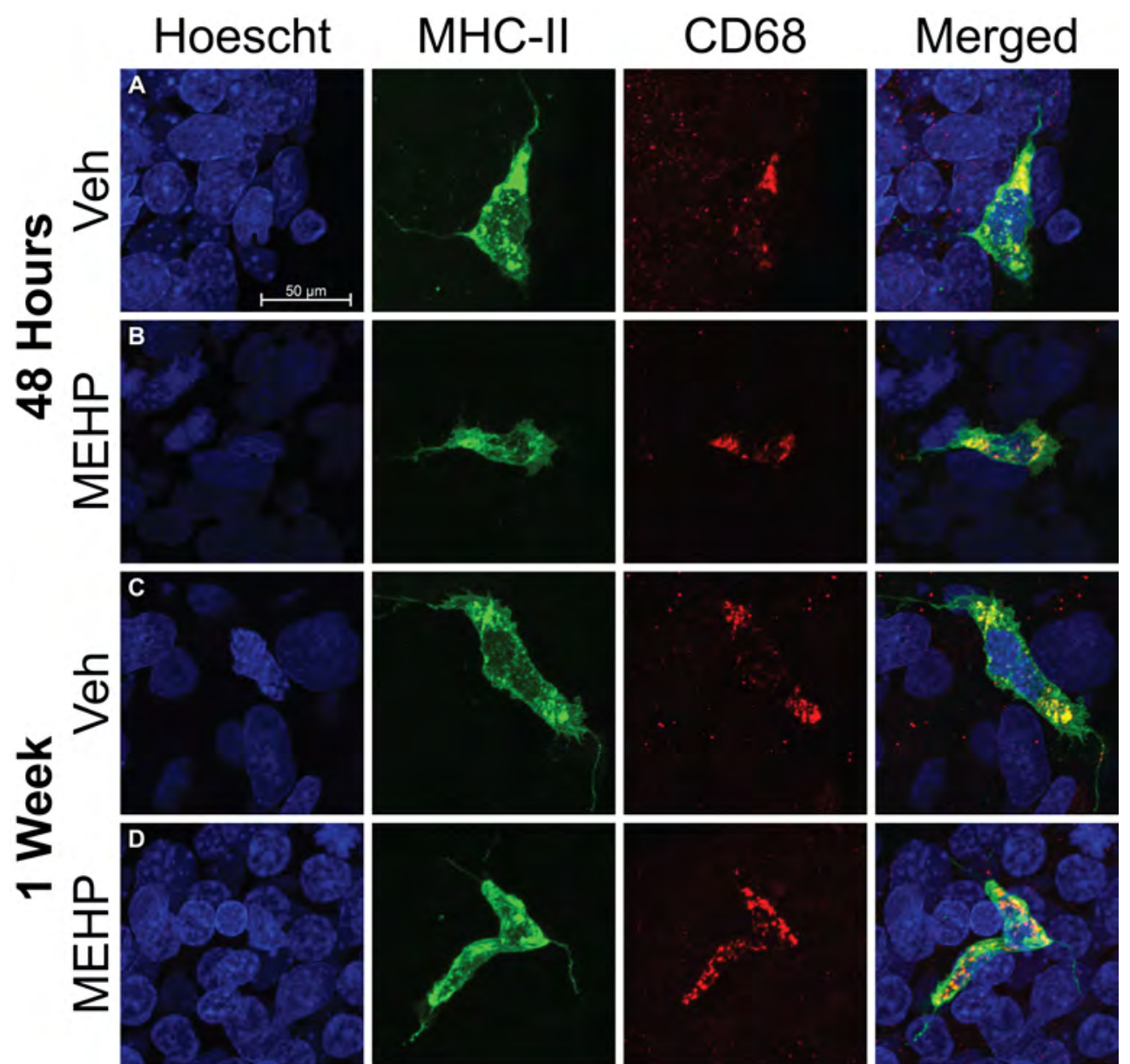

469
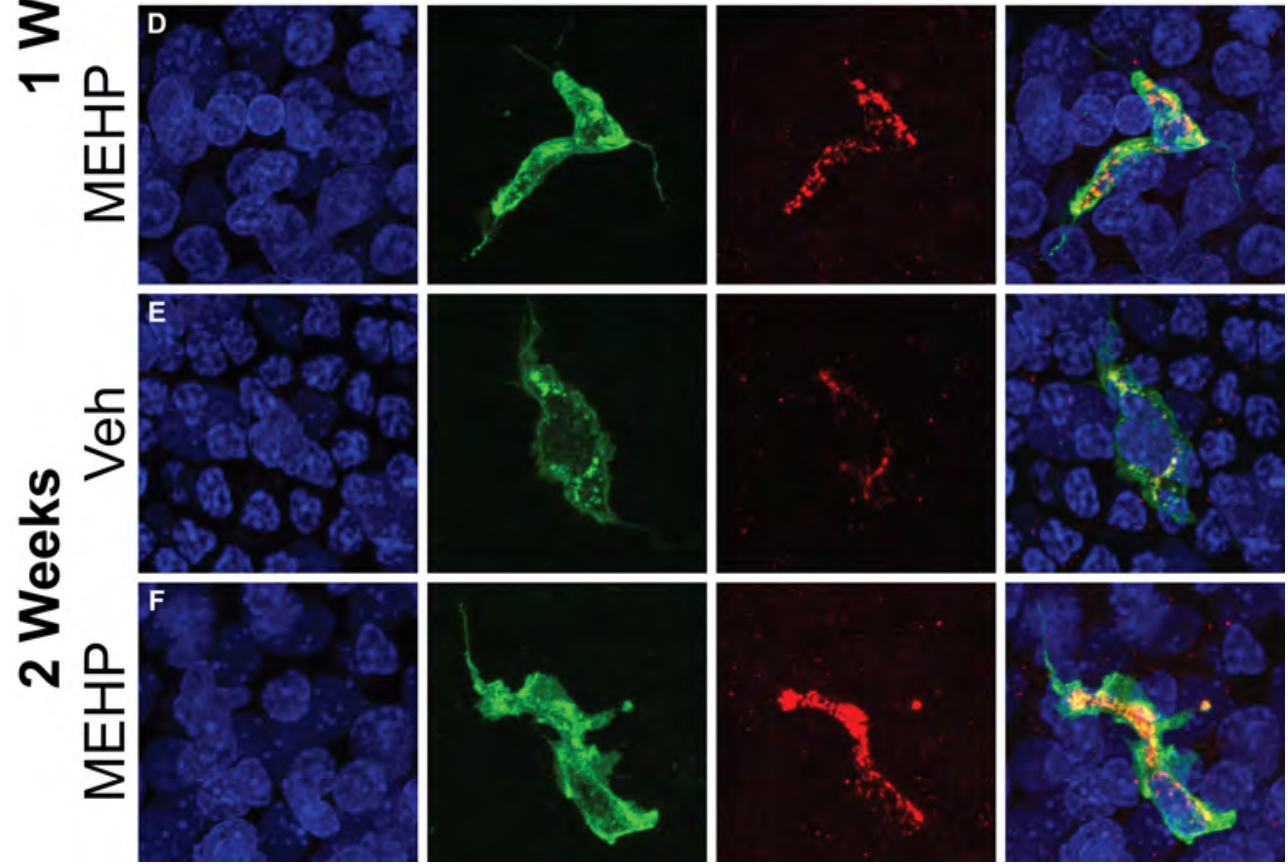

470
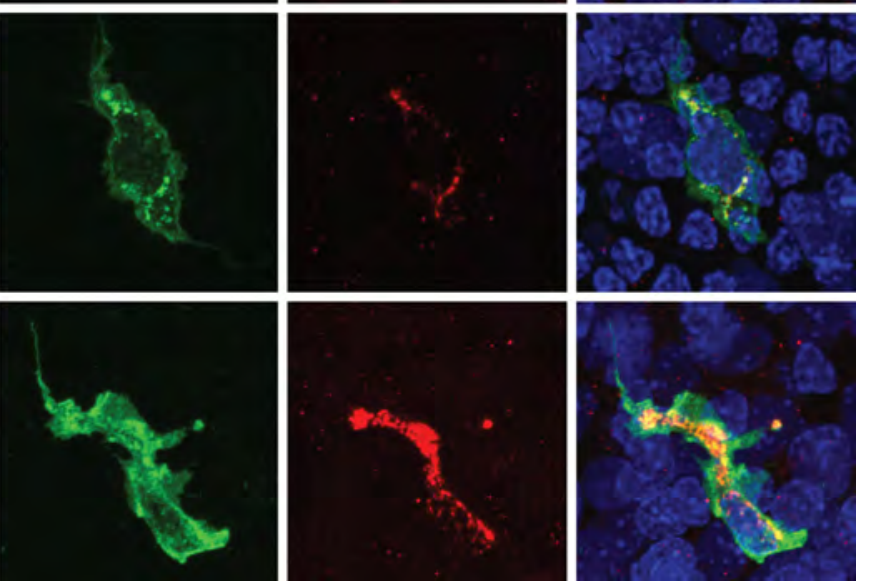
$471 \quad$ Figure 5

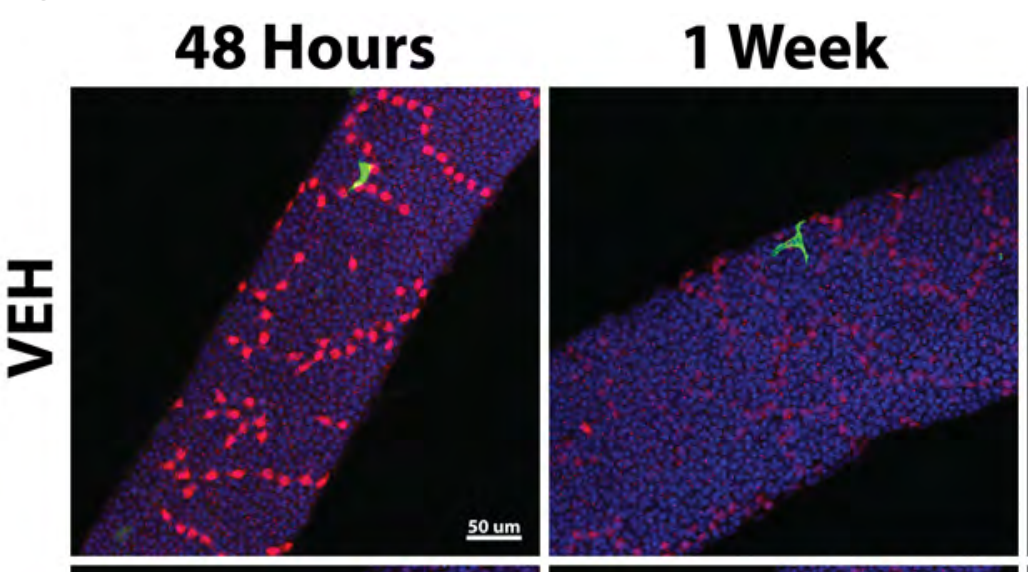

48 Hours
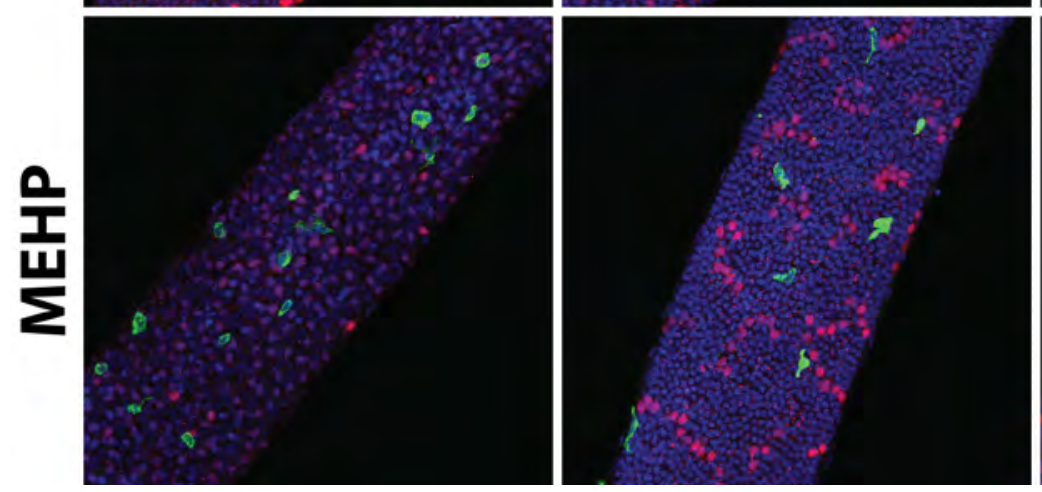

2 Weeks
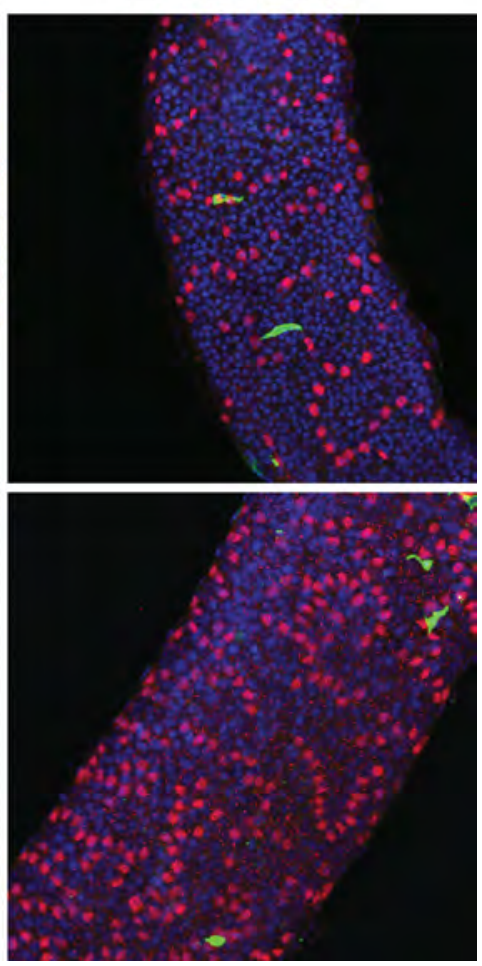


\section{$474 \quad$ Figure 6}

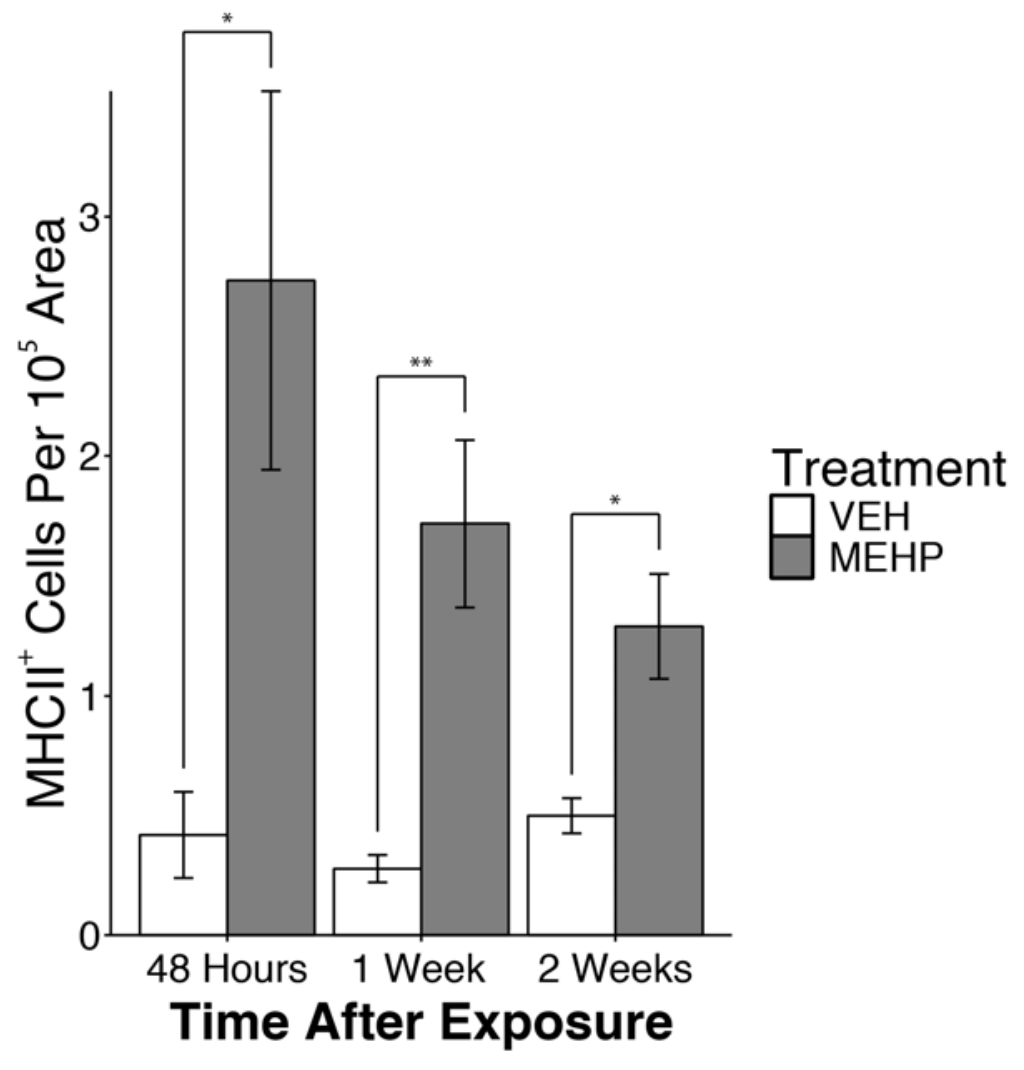


$477 \quad$ Figure 7

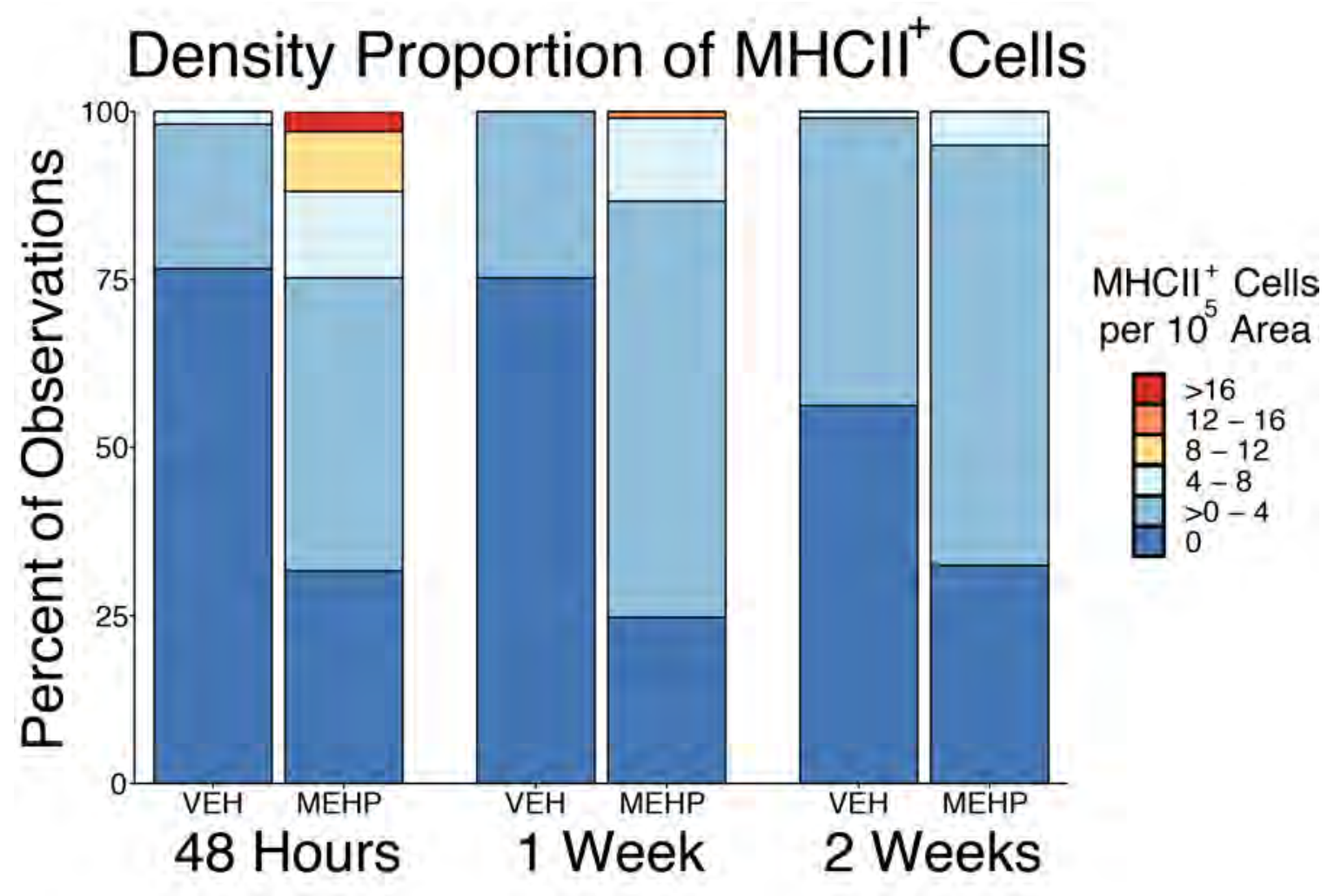




\section{Figure 8}

481

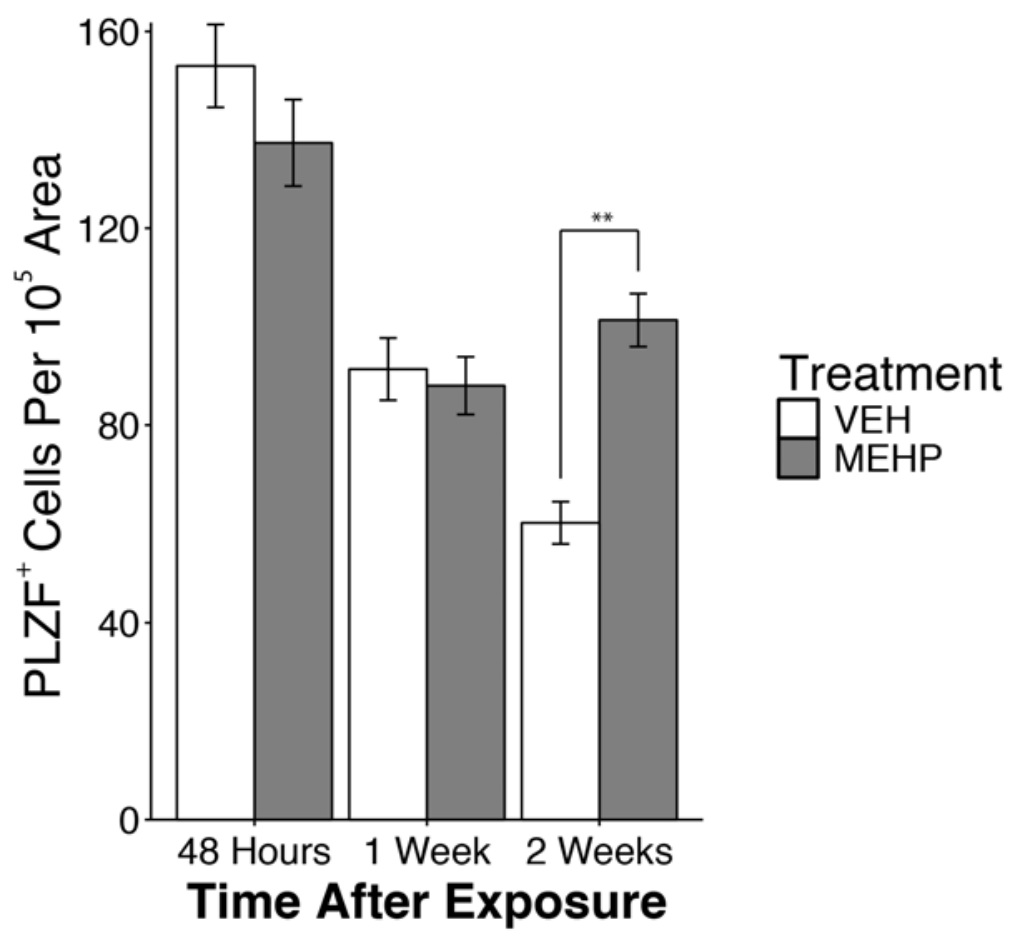

